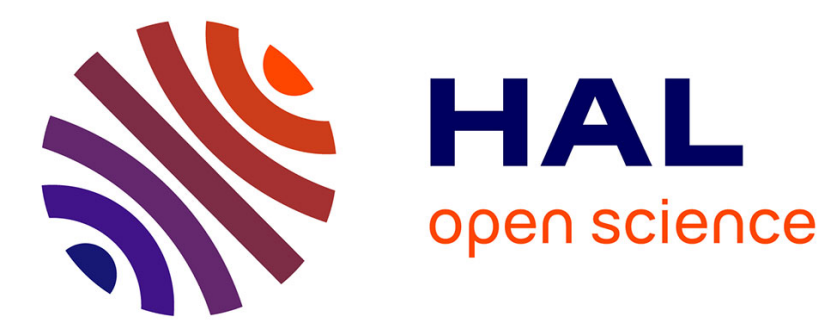

\title{
A new mixed wheel slip and acceleration control based on a cascaded design
}

William Pasillas-Lépine, Antonio Loria

\section{To cite this version:}

William Pasillas-Lépine, Antonio Loria. A new mixed wheel slip and acceleration control based on a cascaded design. NOLCOS 2010, Sep 2010, Bologne, Italy. pp.ThM05.2, 10.3182/20100901-3-IT2016.00231 . hal-00521526

\section{HAL Id: hal-00521526 \\ https://hal-centralesupelec.archives-ouvertes.fr/hal-00521526}

Submitted on 10 Jun 2013

HAL is a multi-disciplinary open access archive for the deposit and dissemination of scientific research documents, whether they are published or not. The documents may come from teaching and research institutions in France or abroad, or from public or private research centers.
L'archive ouverte pluridisciplinaire HAL, est destinée au dépôt et à la diffusion de documents scientifiques de niveau recherche, publiés ou non, émanant des établissements d'enseignement et de recherche français ou étrangers, des laboratoires publics ou privés. 


\title{
A new mixed wheel slip and acceleration control based on a cascaded design
}

\author{
William Pasillas-Lépine and Antonio Loría \\ CNRS (Centre national de la recherche scientifique) \\ L2S - SUPELEC - Univ Paris-Sud \\ 3 rue Joliot-Curie, 91192 Gif-sur-Yvette cedex (France) \\ E-mail: [pasillas,loria]@lss.supelec.fr
}

\begin{abstract}
In this paper, a new cascaded wheel-slip control strategy based on wheel slip and wheel acceleration measurements is presented. This new algorithm is able to stabilize globally and asymptotically the wheel slip around any prescribed setpoint, both in the stable and unstable regions of the tyre.
\end{abstract}

Keywords: Anti-lock braking systems, wheel slip control, cascaded design.

\section{INTRODUCTION}

\subsection{Anti-lock brake systems}

The purpose of anti-lock brake systems (ABS) is twofold. On the one hand, their objective is to avoid wheel lock-up (in order to preserve the tyre ability of producing a lateral force, and thus vehicle maneuverability). On the other hand, they try to keep the wheel slip in a neighborhood of the point that maximizes tyre force (in order to minimize the vehicle's braking distance). In both cases, the specified objective can be reached if we are able to regulate wheel slip in the neighborhood of an appropriate setpoint.

The ABS regulation logic is usually based on several measures. The main source of information is the measure of the angular velocity of the vehicle's wheel that is currently being controlled. But other informations might be available (like the speed of the vehicle or the angular acceleration of the wheel), depending on the kind of vehicle on which the system is embedded (automobile, motorcycle, airplane, etc.).

\subsection{State of the art}

In the literature, one can mainly find two different kinds of anti-lock brake system designs: those based on logic switching from wheel deceleration information (see e.g. Kiencke and Nielsen (2000), Pasillas-Lépine (2006), and Robert Bosch GmbH (2003)) and those based on wheel slip regulation (see e.g. Johansson and Rantzer (2003), Petersen et al. (2001), Tanelli et al. (2008)). There is, however, a third kind of algorithms that use both wheel slip and wheel acceleration measurements Savaresi et al. (2007).

Anti-lock brake strategies based only on wheel deceleration information have quite interesting properties. Indeed, these strategies are very robust with respect to friction coefficient changes and can keep the wheel slip in a neighborhood of the optimal point, without using explicitly the value of the optimal setpoint. But a par- ticularly unpleasant characteristic of these approaches is that they are often based on heuristic arguments, and thus tuning the thresholds involved in this kind of algorithms might be a difficult task Kiencke and Nielsen (2000). Even if some recent results Pasillas-Lépine (2006) give a first step towards a mathematical background for algorithms based on wheel deceleration thresholds (by an analysis of the stability of their limit cycles), these algorithms can only be used in order to track the optimal value of wheel slip $\lambda_{0}$. Namely, the value for which $\mu^{\prime}\left(\lambda_{0}\right)=0$. In other words, they are not able to stabilize the system around an arbitrary reference $\lambda^{*}$ that belongs to the stable $\left(\lambda^{*}<\lambda_{0}\right)$ or unstable region $\left(\lambda^{*}>\lambda_{0}\right)$ of the tyre.

Approches based on pure wheel slip regulation have also quite interesting properties: the torque applied to the wheel converges to a fixed value (there are no periodic oscillations, like in wheel deceleration based algorithms Gerard et al. (2012)) and they work even if there is no clear maximum in the tyre characteristic. Their usage is nevertheless confronted to two difficulties. Firstly, they are mainly based on linearization arguments. The nonlinear system is linearized around the desired equilibrium point, and the stability analysis is thus only valid locally (see e.g. Petersen et al. (2001) and Savaresi et al. (2007)). Secondly, they might fail to work in the unstable region of the tyre (see e.g. Tanelli et al. (2008), where the control strategy generates a limit cycle if the setpoint is in the unstable domain). Thirdly, the available approaches are mainly based on pure feedback (there are no feedforward terms), which considerably limits the bandwith of the closed-loop system.

\subsection{Our contribution}

In this paper, a new cascaded wheel-slip control strategy based on wheel slip and wheel acceleration measurements is presented. This new algorithm is able to stabilize globally and asymptotically the wheel slip around any prescribed setpoint, both in the stable and unstable regions of the tyre. Moreover, it gives precise bounds on the gains of the control law for which stability is proved 
mathematically. Another original point of our approach is the feedforward term, which allows us to improve the bandwith of the regulation scheme.

\section{WHEEL DYNAMICS}

\subsection{Wheel and tyre modeling}

The angular velocity $\omega$ of a given wheel of the vehicle has the following dynamics:

$$
I \frac{d \omega}{d t}=-R F_{x}+T
$$

where $I$ denotes the inertia of the wheel, $R$ its radius, $F_{x}$ the longitudinal tyre force, and $T$ the torque applied to the wheel.

The longitudinal tyre force $F_{x}$ is modelled by a relation

$$
F_{x}\left(\lambda, F_{z}\right)=\mu(\lambda) F_{z} .
$$

That is, by a function that depends linearly on vertical load $F_{z}$ and nonlinearly on the variable

$$
\lambda=\frac{R \omega-v_{x}}{v_{x}},
$$

which is called wheel slip. In this expression $v_{x}$ denotes the longitudinal speed of the vehicle. It should be noted that this definition of slip shows a singularity at zero vehicle speed.

The nondimensional tyre characteristic $\mu(\cdot)$ is a skewsymmetric bounded curve, such that

$$
\mu(0)=0 \text { and } \mu^{\prime}(0)>0 .
$$

One of the simplest models for such a curve is given by

$$
\mu(\lambda)=D \sin (C \arctan (B \lambda)),
$$

which is a simplified form of Pacejka's magic formula (Pacejka, 2006). The three coefficients $B, C$, and $D$ are positive.

The tyre load $F_{z}$ will be assumed to be constant and the vehicle will be supposed to brake with a deceleration $a_{x}(t)$. That is

$$
\frac{d v_{x}}{d t}=a_{x}(t)
$$

In simulations with a quarter-car vehicle model, we will take $a_{x}(t)=\mu(\lambda) g$, where $g$ denotes the gravity.

\subsection{State-space variables of the control system}

If we define the variables $x_{1}$ and $x_{2}$ by

$$
\begin{aligned}
& x_{1}=\lambda \\
& x_{2}=R \frac{d \omega}{d t}-a_{x}(t),
\end{aligned}
$$

then we have the following dynamics

$$
\begin{aligned}
& \frac{d x_{1}}{d t}=\frac{1}{v_{x}(t)}\left(-a_{x}(t) x_{1}+x_{2}\right) \\
& \frac{d x_{2}}{d t}=-\frac{a \mu^{\prime}\left(x_{1}\right)}{v_{x}(t)}\left(-a_{x}(t) x_{1}+x_{2}\right)+\frac{u}{v_{x}(t)}-\frac{d a_{x}}{d t}
\end{aligned}
$$

where

$$
a=\frac{R^{2}}{I} F_{z} \quad \text { and } \quad u=v_{x}(t) \frac{R}{I} \dot{T} .
$$

Observe that we consider as a control variable the derivative of the torque applied to the wheel ; not the torque itself. Depending on the kind of technology used by the brake actuator (EMB or EHB), it might be necessary to integrate the control in order to have a brake torque reference.

For a given time-dependent wheel-slip reference $\lambda^{*}(t)$, we will define a filtered setpoint

$$
\begin{aligned}
\frac{d \lambda_{1}}{d t} & =\frac{\lambda_{2}}{v_{x}(t)} \\
\frac{d \lambda_{2}}{d t} & =\frac{-\gamma_{1}\left(\lambda_{1}-\lambda^{*}\right)-\gamma_{2} \lambda_{2}}{v_{x}(t)} .
\end{aligned}
$$

They aim of this set-point filter is twofold. On the one hand, it allows to have a smooth set-point (that one can differentiate twice) event if the original set-point is discontinuous (for exemple, piecewise constant). On the other hand, it allows to have a system for which all equations are divided by the vehicle's velocity. This homogeneity will allow us to analyse the system in a new (nonlinear) time-scale in which the dependence on speed disappears.

For further development we assume that $a_{x}$ is constant and we apply a change of time-scale as in Pasillas-Lépine (2006). Let

$$
s(t):=\int_{0}^{t} \frac{d \tau}{v_{x}(\tau)}
$$

hence, $d t=v_{x}(t) d s$ and consequently, for any function $\varphi: \mathbb{R} \rightarrow \mathbb{R}^{n}$ we have

$$
\frac{d \varphi}{d s}=\frac{d \varphi}{d t} \frac{d t}{d s}
$$

Therefore, defining $\dot{\varphi}(s)=\frac{d \varphi(s)}{d s}$ we have

$$
\begin{aligned}
& \dot{x}_{1}=-a_{x} x_{1}+x_{2} \\
& \dot{x}_{2}=-a \mu^{\prime}\left(x_{1}\right)\left[-a_{x} x_{1}+x_{2}\right]+u \\
& \dot{\lambda}_{1}=\lambda_{2} \\
& \dot{\lambda}_{2}=-\gamma_{1}\left(\lambda_{1}-\lambda^{*}\right)-\gamma_{2} \lambda_{2} .
\end{aligned}
$$

In the sequel, all derivatives are considered with respect to the scaled time and, with an abuse of notation we use the variable $t$ to denote it.

\section{CONTROL DESIGN}

\subsection{Set-point control}

Assume that $\lambda^{*}$ is constant. Let $x_{1}^{*}=\lambda_{1}$ be the desired operating point for $x_{1}$, and define the error coordinates

$$
z_{1}:=x_{1}-x_{1}^{*} \quad \text { and } \quad z_{2}:=x_{2}-x_{2}^{*},
$$

where $x_{2}^{*}$ is to be defined. Let $x_{2}$ be a virtual control input in Equation (2a). Take $\alpha>0$. If $x_{2}=a_{x} x_{1}+\dot{x}_{1}^{*}-\alpha z_{1}$ then, the closed-loop equation for $z_{1}$ reads

$$
\dot{z}_{1}=-\alpha z_{1}+z_{2}
$$

which is exponentially stable if $z_{2}=0$. Hence, for $x_{2}$, we define the desired operating point as

$$
x_{2}^{*}:=a_{x} x_{1}+\dot{x}_{1}^{*}-\alpha z_{1}
$$

and design the control $u$ so that $x_{2} \rightarrow x_{2}^{*}$ asymptotically. To that end, we compute the error dynamics for $\dot{z}_{2}$. 


$$
\begin{aligned}
\dot{z}_{2}= & \dot{x}_{2}-\dot{x}_{2}^{*} \\
= & -a \mu^{\prime}\left(x_{1}\right)\left[-a_{x} x_{1}+x_{2}\right]+u-\left(a_{x} \dot{x}_{1}+\ddot{x}_{1}^{*}-\alpha \dot{z}_{1}\right) \\
= & -a \mu^{\prime}\left(x_{1}\right)\left[-a_{x} x_{1}+x_{2}\right]+u-a_{x}\left[-a_{x} x_{1}+x_{2}\right]-\dot{\lambda}_{2} \\
& +\alpha\left[-\alpha z_{1}+z_{2}\right] \\
= & -\left[a \mu^{\prime}\left(x_{1}\right)+a_{x}\right]\left[-a_{x} x_{1}+x_{2}\right]-\alpha^{2} z_{1}+\alpha z_{2}-\dot{\lambda}_{2}+u \\
& {\left[\operatorname{using}-a_{x} x_{1}+x_{2}=-\alpha z_{1}+z_{2}+\lambda_{2}\right] } \\
= & \alpha z_{1}\left[a \mu^{\prime}\left(x_{1}\right)+a_{x}-\alpha\right]-z_{2}\left[a \mu^{\prime}\left(x_{1}\right)+a_{x}-\alpha\right] \\
& -\left[a \mu^{\prime}\left(x_{1}\right)+a_{x}\right] \lambda_{2}-\dot{\lambda}_{2}+u .
\end{aligned}
$$

Hence, defining $\eta\left(x_{1}\right):=a \mu^{\prime}\left(x_{1}\right)+a_{x}-\alpha$ and $\psi\left(x_{1}\right):=$ $-a \mu^{\prime}\left(x_{1}\right)-a_{x}$ we obtain

$$
\dot{z}_{2}=\eta\left(x_{1}\right)\left[\alpha z_{1}-z_{2}\right]+\psi\left(x_{1}(t)\right) \lambda_{2}+\dot{\lambda}_{2}+u .
$$

The wheel-slip control problem comes to stabilizing the system (4), (6) at the origin.

Our first controller is the simple linear Proportional Derivative controller ${ }^{1}$

$$
u=-k_{1} z_{1}-k_{2} z_{2}
$$

which leads to the following result.

Proposition 1. Assume that there exists a number $c_{\mu}^{M}$ such that

$$
\left|\mu^{\prime}(s)\right| \leq c_{\mu}^{M}, \quad \forall s \in \mathbb{R} .
$$

Then, when $\lambda^{*}$ is constant, the origin $\left(z_{1}, z_{2}\right)=(0,0)$ of the closed-loop system (2) with (5) and (7) is globally exponentially stable for sufficiently large values of $k_{1}$ and $k_{2}$.

Proof. Define $\tilde{\lambda}_{1}:=\lambda_{1}-\lambda^{*}$. Then, closed-loop equations are

$$
\begin{aligned}
\dot{z}_{1}= & -\alpha z_{1}+z_{2} \\
\dot{z}_{2}= & -\left[k_{1}-\alpha \eta\left(x_{1}(t)\right)\right] z_{1}-\left[k_{2}+\eta\left(x_{1}(t)\right)\right] z_{2}+ \\
& +\psi\left(x_{1}\right) \lambda_{2}+\dot{\lambda}_{2} \\
\dot{\tilde{\lambda}}_{1}= & \lambda_{2} \\
\dot{\lambda}_{2}= & -\gamma_{1} \tilde{\lambda}_{1}-\gamma_{2} \lambda_{2}
\end{aligned}
$$

Defining $z:=\left[z_{1} ; z_{2}\right]$ and $\Lambda:=\left[\tilde{\lambda}_{1} ; \lambda_{2}\right]$ we see that the closed-loop system has a so-called cascaded form

$$
\begin{aligned}
& \dot{z}=F_{1}(t, z)+G(t) \Lambda \\
& \dot{\Lambda}=F_{2}(\Lambda)
\end{aligned}
$$

where

$$
\begin{gathered}
F_{1}(t, z):=\left[\begin{array}{c}
-\alpha z_{1}+z_{2} \\
-\left[k_{1}-\alpha \eta\left(x_{1}(t)\right)\right] z_{1}-\left[k_{2}+\eta\left(x_{1}(t)\right)\right] z_{2}
\end{array}\right], \\
F_{2}(\Lambda):=\left[\begin{array}{cc}
0 & 1 \\
-\gamma_{1} & -\gamma_{2}
\end{array}\right],
\end{gathered}
$$

and

$$
G(t):=\left[\begin{array}{cc}
0 & 0 \\
-\gamma_{1} & \psi\left(x_{1}(t)\right)-\gamma_{2}
\end{array}\right] .
$$

According to results for cascaded systems the system (10) is globally exponentially stable at the origin if:

\footnotetext{
1 In view of (1), Eq. (7) is implemented by using $\dot{T}=\frac{u I}{R v_{x}}$.
}

a) the origin of $\dot{z}=F_{1}(t, z)$ is globally exponentially stable;

b) the origin of $\dot{\Lambda}=F_{2}(\Lambda)$ is globally exponentially stable;

c) the solutions are globally bounded uniformly in the initial conditions.

The second condition evidently holds for any positive values of $\gamma_{1}, \gamma_{2}$ as the system is linear time-invariant and of second order. The third condition holds if $G(t)$ is bounded uniformly for all $t$ (see Loria and Panteley, 2005). The latter holds ${ }^{2}$, since $\psi\left(x_{1}\right)$ is bounded for all $x_{1} \in \mathbb{R}$.

It is only left to show that the origin of $\dot{z}=F_{1}(t, z)$ is globally exponentially stable. We proceed using standard Lyapunov theory. Define

$$
\begin{aligned}
\kappa_{1}\left(x_{1}\right) & :=k_{1}-\alpha \eta\left(x_{1}\right) \\
\kappa_{2}\left(x_{1}\right) & :=k_{2}+\eta\left(x_{1}\right) .
\end{aligned}
$$

The total time derivative of the Lyapunov function

$$
V\left(z_{1}, z_{2}\right):=\varepsilon z_{1}^{2}+\frac{1}{2} z_{2}^{2}
$$

along the closed-loop trajectories generated by $\dot{z}=F_{1}(t, z)$ yields

$$
\dot{V}\left(z_{1}, z_{2}\right)=z_{2}\left[-\kappa_{1}\left(x_{1}\right) z_{1}-\kappa_{2}\left(x_{1}\right) z_{2}\right]+\varepsilon\left(-\alpha z_{1}+z_{2}\right) z_{1}
$$

which is equivalent to

$$
\begin{aligned}
\dot{V}\left(z_{1}, z_{2}\right)= & -\frac{1}{2}\left[\begin{array}{c}
z_{1} \\
z_{2}
\end{array}\right]^{\top}\left[\begin{array}{cc}
\varepsilon \alpha & \kappa_{1}\left(x_{1}\right)-\varepsilon \\
\kappa_{1}\left(x_{1}\right)-\varepsilon & \kappa_{2}\left(x_{1}\right)
\end{array}\right]\left[\begin{array}{l}
z_{1} \\
z_{2}
\end{array}\right] \\
& -\frac{1}{2} \varepsilon \alpha z_{1}^{2}-\frac{1}{2} \kappa_{2}\left(x_{1}\right) z_{2}^{2}
\end{aligned}
$$

The matrix in (12) is positive semidefinite if

which holds if

$$
\varepsilon \alpha\left[k_{2}+\eta\left(x_{1}\right)\right] \geq\left[k_{1}-\alpha \eta\left(x_{1}\right)-\varepsilon\right]^{2}
$$

$$
\varepsilon \alpha\left[k_{2}+\eta\left(x_{1}\right)\right] \geq 2\left[\left(k_{1}-\varepsilon\right)^{2}+\alpha^{2} \eta\left(x_{1}\right)^{2}\right] .
$$

In view of (8) there exist positive constants $\eta_{m}$ and $\eta_{M}$ such that $\eta_{M} \geq \eta\left(x_{1}\right) \geq-\eta_{m}$ hence (13) holds if

$$
\varepsilon \alpha\left(k_{2}-\eta_{m}\right) \geq 2\left[\left(k_{1}-\varepsilon\right)^{2}+\alpha^{2} \eta_{M}^{2}\right]
$$

or equivalently, if for any $k_{1} \geq 0$

$$
k_{2} \geq \frac{2\left[\left(k_{1}-\varepsilon\right)^{2}+\alpha^{2} \eta_{M}^{2}\right]}{\varepsilon \alpha}+\eta_{m} .
$$

In particular, for $\varepsilon=\alpha$ it holds if

$$
k_{2} \geq 2\left[\frac{k_{1}^{2}}{\alpha^{2}}-\frac{2 k_{1}}{\alpha}+\eta_{M}^{2}+1\right]+\eta_{m} .
$$

Under the latter conditions we have

$$
\dot{V} \leq-\frac{1}{2} \alpha^{2} z_{1}^{2}-\kappa_{2 m} z_{2}^{2}
$$

where $\kappa_{2 m}>0$ since $k_{2}+\eta\left(x_{1}\right) \geq k_{2}-\eta_{m}>0$. The result follows from standard Lyapunov theory.

Following up the previous proof we may compute convenient explicit exponential bound for the error trajectories. To that end, let $\kappa_{2 m} \geq \alpha / 4$ and define $v(t):=$ $V\left(z_{1}(t), z_{2}(t)\right)$. We have

$$
\dot{v}(t) \leq-\frac{\alpha}{2} v(t)
$$

\footnotetext{
2 Strictly speaking one needs to show that the trajectories $x_{1}(t)$ exist for all $t$ but we shall not dwell with that here and (safely) assume that it is the case.
} 
hence, integrating on both sides from 0 to $t$

$$
v(t) \leq v(0) e^{-\frac{\alpha}{2} t} \leq v(0)
$$

and using the definition of $V$ we obtain

$$
\min \left\{\frac{1}{2}, \alpha\right\}\left[\left|z_{1}(t)\right|^{2}+\left|z_{2}(t)\right|^{2}\right] \leq v(t) \leq v(0),
$$

with

$$
v(0) \leq \max \left\{\frac{1}{2}, \alpha\right\}\left[\left|z_{1}(0)\right|^{2}+\left|z_{2}(0)\right|^{2}\right] .
$$

Finally, from (17), (18), and (19), we see that

$$
|z(t)| \leq \sqrt{\frac{\min \left\{\frac{1}{2}, \alpha\right\}}{\max \left\{\frac{1}{2}, \alpha\right\}}}|z(0)| e^{-\frac{\alpha}{4} t}
$$

That is, both the convergence rate and overshoot are proportional to the design parameter $\alpha$.

\subsection{Feedforward \& Adaptation}

From a systems analysis viewpoint the terms $\psi\left(x_{1}\right) \lambda_{2}+\dot{\lambda}_{2}$ are considered as a (vanishing) perturbation to the first two stabilizing terms in Equation (9b). It is an elementary fact that exponential systems are robust to vanishing perturbations and therefore, the stability is unaffected under the influence of $\psi\left(x_{1}\right) \lambda_{2}+\dot{\lambda}_{2}$. This is formally showed in the previous proof.

However, even though the linear controller (7) ensures global exponential stability it may be expected that it yields a relatively poor transient performance; this may actually be appreciated in the Simulations. It takes a simple modification to construct the Proportional Derivative plus Feedforward compensation controller

$$
u=-k_{1} z_{1}-k_{2} z_{2}+v
$$

with

$$
v=\psi\left(x_{1}\right) \lambda_{2}+\dot{\lambda}_{2}
$$

Then, the closed-loop equations (9a), (9b) become $\dot{z}=$ $F_{1}(t, z)$ for which we have showed that the origin is globally exponentially stable. The result of Proposition 1 holds.

Let the function $\mu$ be composed of a linear combination of $N$ generating functions $\varphi_{i}$ with unknown coefficients. More precisely, let its derivative have the form

$$
\mu^{\prime}\left(x_{1}\right):=\sum_{i=1}^{N} \theta_{i} \varphi_{i}\left(x_{1}\right)=\Theta^{\top} \Phi\left(x_{1}\right) .
$$

where the vector of constant parameters $\Theta$ is unknown. Then, we can make the following statement.

Proposition 2. Consider the system (2) in closed loop with

$$
\begin{aligned}
& u=-k_{1} z_{1}-k_{2} z_{2}+\hat{v} \\
& \hat{v}=\left[\hat{\Theta}^{\top} \Phi\left(x_{1}\right) a+a_{x}\right] \lambda_{2}+\dot{\lambda}_{2} \\
& \dot{\hat{\Theta}}=-\gamma z_{2} \Phi\left(x_{1}\right) a
\end{aligned}
$$

and let the control gains satisfy the conditions from Proposition 1. Then, the closed-loop trajectories satisfy

$$
\left|z_{2}(t)\right| \rightarrow 0, \quad\left|z_{1}(t)\right| \rightarrow 0, \quad \text { as } t \rightarrow \infty
$$

and the origin of system $(9),(z, \Lambda)=(0,0)$ is uniformly globally stable that is, the trajectories are uniformly globally bounded and the origin is uniformly stable.
Proof. Let the estimation error be denoted by $\tilde{\Theta}:=\hat{\Theta}-\Theta$ and let us define $\tilde{v}:=\hat{v}-v^{*}$ where $v^{*}$ corresponds to the feedforward terms on the right-hand side of (22)i.e., in the case that the parameters $\Theta$ are known:

$$
v^{*}=\left[\Theta^{\top} \Phi\left(x_{1}\right) a+a_{x}\right] \lambda_{2}+\dot{\lambda}_{2} .
$$

With these notations, we see that (23) can be written as

$$
u=-k_{1} z_{1}-k_{2} z_{2}+v^{*}+\tilde{v} .
$$

Substituting this in (6) we see that the closed-loop equation becomes

$$
\dot{z}_{2}=-\left[k_{1}-\alpha \eta\left(x_{1}\right)\right] z_{1}-\left[k_{2}+\eta\left(x_{1}\right)\right] z_{2}+\tilde{\Theta}^{\top} \Phi\left(x_{1}\right) a
$$
where we used $\tilde{v}=\tilde{\Theta}^{\top} \Phi\left(x_{1}\right) a$.

Now, define $V_{1}:=V$ with $V$ as in (11). After the proof of Proposition 1 the time derivative of $V$ along the closedloop trajectories generated by $(9 \mathrm{a}),(26)$ yields

$$
\dot{V}_{1} \leq-\frac{1}{2} \alpha^{2} z_{1}^{2}-\kappa_{2 m} z_{2}^{2}+z_{2} \tilde{\Theta}^{\top} \Phi\left(x_{1}\right) a
$$

Let

$$
V_{2}:=\frac{1}{2 \gamma}|\tilde{\Theta}|^{2} .
$$

Considering that $\Theta$ is constant the time derivative of $V_{2}$ along the trajecotires generated by the adaptation law (25) yields

$$
\dot{V}_{2}=-z_{2} \tilde{\Theta}^{\top} \Phi\left(x_{1}\right) a .
$$

Therefore the time derivative of $V:=V_{1}+V_{2}$ along the closed loop trajectories satisfies

$$
\dot{V} \leq-\frac{1}{2} \alpha^{2} z_{1}^{2}-\kappa_{2 m} z_{2}^{2} \leq 0
$$

The latter and the fact that $V$ is positive definite imply uniform stability of the origin.

Define $\xi:=[z ; \Theta]$. Similarly to $V_{1}$ in (18) it is easy to see that $V(\xi(t)) \geq \min \left\{\frac{1}{2}, \alpha\right\}|\xi(t)|^{2} \geq 0$ and $V(\xi(0)) \leq$ $\max \left\{\frac{1}{2}, \alpha\right\}|\xi(0)|^{2}<\infty$ Then, integrating on both sides of the first inequality in (28) from 0 to $\infty$ we obtain, on one hand,

$$
\min \left\{\frac{1}{2}, \alpha\right\} \int_{0}^{\infty}|z(t)|^{2} d t \leq \max \left\{\frac{1}{2}, \alpha\right\}\left[|\xi(0)|^{2}\right]
$$

and on the other, integrating on both sides of the second inequality i.e. of $\dot{V} \leq 0$ we obtain

$$
\min \left\{\frac{1}{2}, \alpha\right\}|\xi(t)|^{2} \leq \max \left\{\frac{1}{2}, \alpha\right\}|\xi(0)|^{2}
$$

that is, the error trajectories are uniformly globally bounded and so is $x_{1}(t):=z_{1}(t)+\lambda_{1}(t)$ since $\lambda_{1}(t) \rightarrow 0$ as $t \rightarrow \infty$.

Finally, using (29) in (26), (25) and (9a) we see that there exists a continuous function $\beta$ such that

$$
\max \left\{\sup _{t \geq 0}|\dot{z}(t)|, \sup _{t \geq 0}|\dot{\tilde{\Theta}}(t)|\right\} \leq \beta(|\xi(0) ; \lambda(0)|) .
$$

The result follows applying Lemma 4 , given below.

Remark 3. It is important to stress that, in general the solutions are bounded uniformly by a number that depends on the size of initial conditions $z(0), \tilde{\Theta}(0)$ and $\lambda(0)$.

The following statement Kelly et al. (2005) is an adaptation of Barbalat's Lemma to avoid the condition "if the limit exists and is finite ..." imposed in Barbalat's lemma. The advantage is that the conditions of the lemma below are exactly those we show in the proof. 
Lemma 4. Consider a once continuously differentiable function $f: \mathbb{R}_{+} \rightarrow \mathbb{R}^{m}$. Suppose that there exist constants $c_{1}$ and $c_{2}$ such that $f$ and its time derivative satisfy

$$
\begin{gathered}
\max \left\{\sup _{t \geq 0}|f(t)|, \quad \sup _{t \geq 0}|\dot{f}(t)|\right\} \leq c_{1} \\
\int_{0}^{\infty}|f(t)|^{2} d t \leq c_{2}
\end{gathered}
$$

Then,

$$
\lim _{t \rightarrow \infty} f(t)=0
$$

\section{SIMULATION RESULTS}

The simulation model used to test our theoretical results is the simple quarter-car model of Subsection 2.1, in which the control laws of Subsections 3.1 and 3.2 have been injected.

The first aim of our simulations is to observe the effects of the feedback and feedforward terms. In accordance both with intuition and with our theoretical study, the following phenomena can be observed :

a) When the feedback gains are equal to zero (that is, in the case of pure feedforward control), the system tracks the desired wheel slip reference only if this reference is in the tyre's stable zone ; otherwise the purely open-loop system is unstable (Figure 1).

b) When the feedback gains satisfy the conditions given in the proof of Proposition 1 and the feedforward is not used (that is, in the case of pure feedback control), the system tracks the desired wheel slip reference, but with a very poor performance during transients (Figure 2).

c) When both feedback and feedforward terms are included, the system follows exactly the filtered reference (Figure 3). But this, only if there are no perturbations on the system (like delays or uncertainties on the system's parameters).

The second aim of our simulations is to observe the effects of perturbations, in order to evaluate the robustness of our control laws (when both feedback and feedforward terms are used). We considered mainly three cases :

a) When a pure delay is introduced in the control loop (take, for example, the case of an actuator delay) the performance remains good and the system remains stable, provided that the delay is not too big (Figure 4).

b) When the system's parameters used in the control law do not match those of the true system (like, for exemple, a change of tyre characteristics) the system remains stable, but the performance is considerably reduced (Figure 5). Notice that in this simulation, the adaptation law was not implemented.

c) When both pure delay and parameter uncertainties are considered, the results are quite close to the case of pure parameter uncertainties (Figure 6).

Simulations with a more complicated quarter-car model have also been made, in order to prepare the implementation of the proposed algorithms on the TU-Delft Tyre Setup, giving similar results (Pasillas-Lépine et al., 2012).

\section{CONCLUSION}

In this paper, a new cascaded wheel-slip control strategy based on wheel slip and wheel acceleration measurements was presented. It was shown that the proposed algorithm is able to stabilize globally and asymptotically the wheel slip around any prescribed setpoint, both in the stable and unstable regions of the tyre.

Compared to other approches, the main original points of our algorithm are :

a) the outputs that are assumed to be measured (both wheel slip and wheel acceleration),

b) the proof of global stability and the bounds obtained for the feedback gains,

c) the filtered reference (that allows an analysis of the system in a new time-scale) and the feedforward terms.

An other original point is the model used for the control synthesis (with the assumption of a constant acceleration), which was an important step to be able to arrive to our results.

\section{REFERENCES}

Gerard, M., Pasillas-Lépine, W., De Vries, E., and Verhaegen, M. (2012). Improvements to a five-phase ABS algorithm for experimental validation. Vehicle System Dynamics, 50(10), 1585-1611.

Johansson, R. and Rantzer, A. (eds.) (2003). Nonlinear and Hybrid Systems in Automotive Control. SpringerVerlag.

Kelly, R., Santibañez, V., and Loria, A. (2005). Control of robot manipulators in joint space. Advanced textbooks in control engineering. Springer Verlag.

Kiencke, U. and Nielsen, L. (2000). Automotive Control Systems. Springer-Verlag.

Loria, A. and Panteley, E. (2005). Cascaded nonlinear time-varying systems: analysis and design. Lecture Notes in Control and Information Sciences. Springer Verlag.

Pacejka, H. (2006). Tyre and Vehicle Dynamics. Butterworths-Heinemann, London.

Pasillas-Lépine, W. (2006). Hybrid modeling and limit cycle analysis for a class of five-phase anti-lock brake algorithms. Vehicle System Dynamics, 44(2), 173-188.

Pasillas-Lépine, W., Loría, A., and Gerard, M. (2012). Design and experimental validation of a nonlinear wheel slip control algorithm. Automatica, 48(8), 1852-1859.

Petersen, I., Johansen, T.A., Kalkkuhl, J., and Lüdemann, J. (2001). Nonlinear wheel slip control in ABS brakes using gain scheduled constrained LQR. In Proceedings of the European Control Conference, 606-611.

Robert Bosch GmbH (2003). Automotive Handbook. Bentley.

Savaresi, S., Tanelli, M., and Cantoni, C. (2007). Mixed slip-deceleration control in automotive braking systems. ASME Journal of Dynamic Systems, Measurement, and Control, 129(1), 20-31.

Tanelli, M., Savaresi, S., and Astolfi, A. (2008). Robust nonlinear output feedback control for brake by wire control systems. Automatica, 44(4), 1078-1087. 


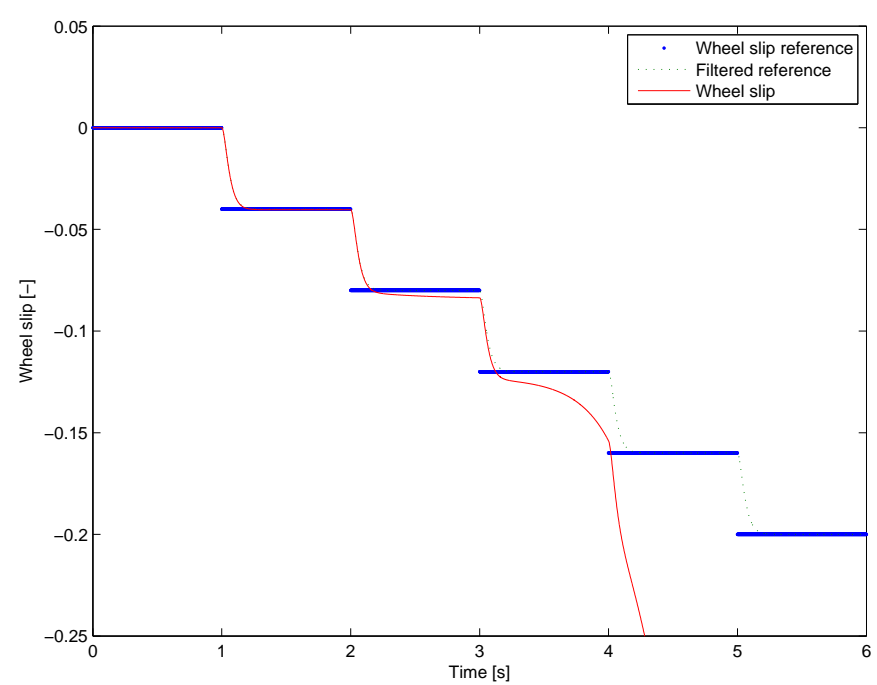

Fig. 1. Pure feedforward control $\left(k_{1}=0\right.$ and $\left.k_{2}=0\right)$.

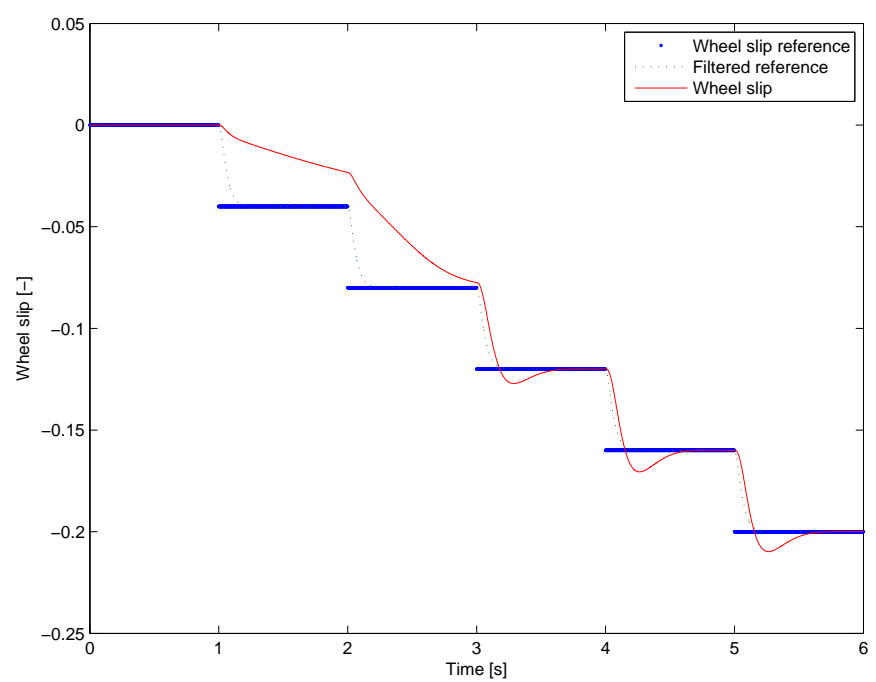

Fig. 2. Pure feedback control.

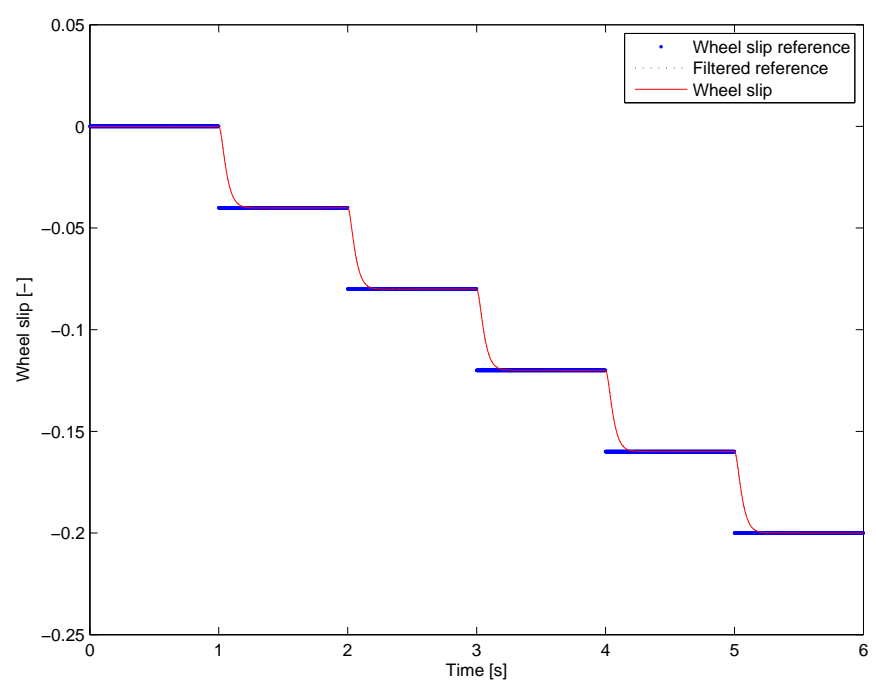

Fig. 3. Combined feedback and feedforward control (without perturbations).

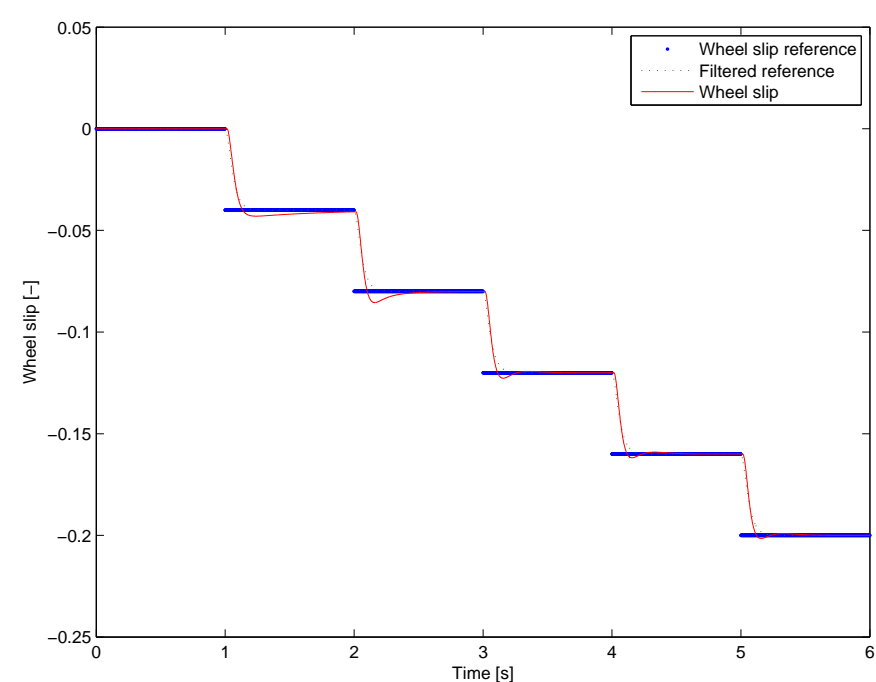

Fig. 4. Combined control, with a delay of $15 \mathrm{~ms}$.

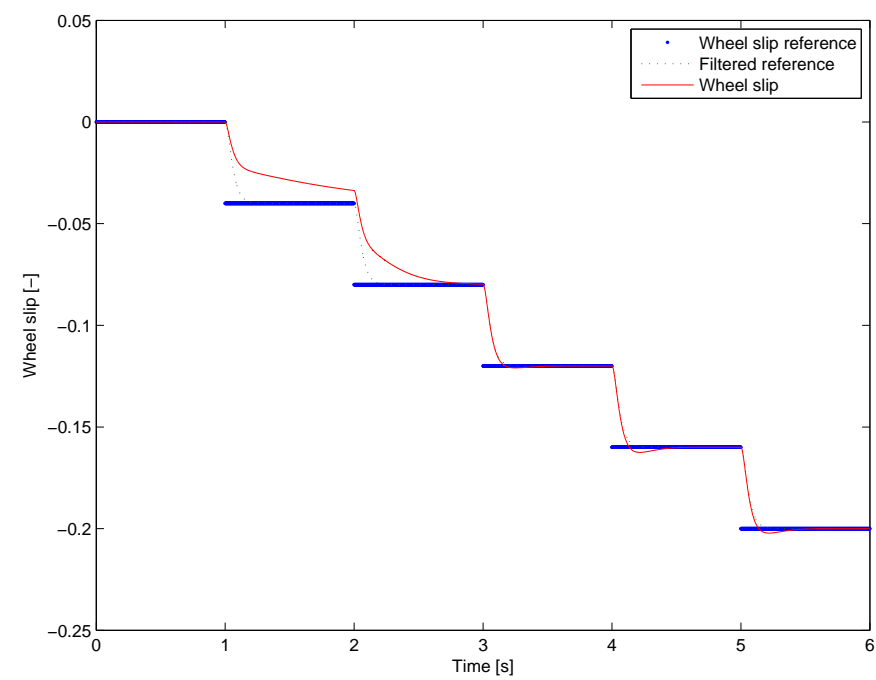

Fig. 5. Combined control with a perturbation of $\mu(\cdot)$.

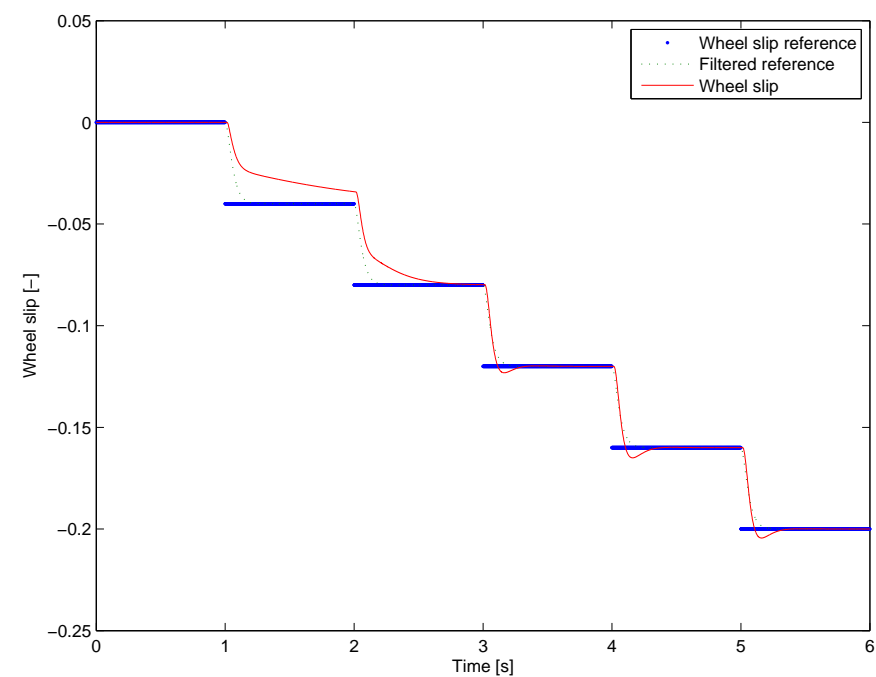

Fig. 6. Combined control, with a delay of $15 \mathrm{~ms}$ and a perturbation of $\mu(\cdot)$. 